\title{
Cardiac damage presenting late after road accidents
}

\author{
ALAN F MACKINTOSH, HUGH A FLEMING \\ From the Regional Cardiac Unit, Papworth Hospital, Cambridge
}

ABSTRACT Six examples of cardiac damage secondary to non-penetrating trauma in road accidents are described. In all six cases the lesion was not recognised at the time of the accident but became clinically important two days to 17 years later. As the patients were young or had unusual lesions, the damage could be attributed to the accident. In older patients with common cardiac problems the trauma might not be recognised as the underlying cause.

Non-penetrating trauma to the chest, often sustained in a road accident, can damage the heart. A wide variety of lesions in the valves, myocardium, coronary arteries, and pericardium has been documented.1-5 Most reports have dealt with necropsy findings or have described cardiac injuries presenting immediately after the accident. In this account six examples of cardiac damage, which was unrecognised at the time of the accident but became clinically important later, are described.

\section{Case reports}

\section{CASE 1}

An 18-year-old man was riding a motor-cycle through a cloud of smoke when he ran into a fire-engine fighting a stubble fire. He sustained multiple injuries including bruising on the left anterior chest wall and a ruptured spleen. No cardiac trauma was detected at the time of admission but two days later he became breathless with pulmonary oedema. An electrocardiogram showed a large transmural anterior infarct. With the help of digoxin and diuretic therapy he made a good recovery. Four years later he is asymptomatic but a third sound and cardiac enlargement persist.

\section{CASE 2}

A man of 46 drove his car into a lamp post. The steering wheel hit his chest and he suffered multiple injuries including a ruptured spleen, a ruptured diaphragm, and rib fractures. After operation he made a good recovery, but tiredness and breathlessness were noticed four months later. He was found to have tricuspid regurgitation, and the electrocardio-

Address for reprint requests: Dr HA Fleming, Regional Cardiac Unit, Papworth Hospital, Papworth Everard, Cambridge CB3 8RE. gram showed right bundle branch block. The only abnormality found at cardiac catheterisation 18 months after the accident was gross tricuspid regurgitation. The peak systolic pressure was $28 \mathrm{~mm} \mathrm{Hg}$ in both the right atrium and the right ventricle. A gradual symptomatic improvement occurred and another catheterisation six months later showed diminished tricuspid regurgitation with a peak systolic pressure of $15 \mathrm{~mm} \mathrm{Hg}$ in the right atrium and $25 \mathrm{~mm} \mathrm{Hg}$ in the right ventricle.

\section{CASE 3}

A 24-year-old man was involved in an accident while riding a motor-cycle. Few details are available, though he sustained multiple injuries. Immediately after the accident his jugular venous pressure was noticed to be raised but no cardiac damage was detected. He was making a good recovery 10 days after the accident when he suddenly became unconscious and developed a right hemiplegia. An electrocardiogram showed a transmural anteroseptal infarct. A diagnosis of a cerebral embolus secondary to a mural thrombus from the infarction site was made. His recovery was only partial and epileptic fits proved difficult to control. He died two years after the accident. Necropsy showed recent and old cerebral infarction secondary to emboli. The coronary arteries were normal apart from complete occlusion of the left anterior descending artery three centimetres from its origin. Microscopy of the occlusion showed periarterial haemosiderosis and breaks in the external elastic lamina; these findings are consistent with traumatic damage to the artery. An old anterior myocardial infarction was probably the source of the recent and old emboli.

CASE 4

In 1941 a 19-year-old man was in a head-on collision 
with a lorry, the steering wheel hitting his chest. Nine years earlier he had been seen by a consultant physician for delayed growth and three months before the accident he had been passed by a medical board for the RAF without any heart disease being detected on either occasion. During his two weeks in hospital after the accident no cardiac murmur was noticed but a systolic murmur was heard five weeks later by his general practitioner. The clinical features of a ventricular septal defect were present and the case was reported soon afterwards. ${ }^{6} \mathrm{He}$ was symptomfree until 1980 when he developed infective endocarditis (Streptococcus viridans), treated with penicillin and gentamicin. Subsequent cardiac catheterisation demonstrated a shunt, apparently at ventricular level, with a pulmonary to systemic flow ratio of $2 \cdot 0: 1$. Surgical repair was advised and at operation (Mr TAH English) three small holes were found between the left ventricle and right atrium. These defects were closed with some difficulty as the conducting system was in close proximity.

CASE 5

At the age of 9 years a boy was run over by a tractor trailer, sustaining injuries to his chest. He made an uncomplicated recovery. Seventeen years later he was found to have constrictive pericarditis with gross pericardial calcification. No history or evidence of tuberculosis could be obtained from the patient or his family. Cardiac catheterisation confirmed the diagnosis and pericardiectomy was performed (Mr BB Milstein) with a good result.

\section{CASE 6}

A 46-year-old taxi-driver drove into a brick wall. He sustained multiple fractures of the right arm, right leg, and ribs. The admission notes recorded the absence of any murmurs. The following day an early diastolic murmur was detected but no attention was paid to it. Two days after admission he developed pulmonary oedema but the possibility of cardiac damage was not considered. His subsequent course was stormy and he needed artificial ventilation for several days. Three weeks after the accident an electrocardiogram showed left ventricular hypertrophy. A diagnosis of aortic regurgitation was made and he was started on therapy for left ventricular failure. When seen for the first time in the cardiac department a year later, he was symptom-free but the chest radiograph showed increasing cardiomegaly. Cardiac catheterisation demonstrated severe aortic regurgitation, and an atrial septal defect with a pulmonary to systemic flow ratio of $2 \cdot 0: 1$. At operation ( $\mathrm{Mr}$ TAH English) fine pericardial adhesions and two posterior pericardial defects were found. The non-coronary cusp of the aortic valve had a large traumatic perforation. The edges of the atrial septal defect were thickened and fibrous; the lesion could only have been produced by trauma. The septal defect was closed and the aortic valve was replaced with a Björk-Shiley prosthesis.

\section{Discussion}

Many examples of cardiac trauma secondary to non-penetrating injury have been described in the last 200 years and the subject has been extensively reviewed. ${ }^{1-5}$ At times certainty that the lesion did not pre-date the injury is missing. Nevertheless cardiac rupture, septal defects, myocardial infarction, coronary artery damage, valve lesions, pericarditis, complete heart block, and many arrhythmias have been described as occurring secondary to nonpenetrating injuries.

The cases described here reflect this diversity of lesions and also contain some unusual features. Case 3 is an example of trauma producing complete occlusion of an otherwise healthy coronary artery. The possibility of such arterial damage has been questioned in the past ${ }^{3}$ and this case is one of the few clear examples. The tricuspid regurgitation found in case 2 is an uncommon traumatic lesion. ${ }^{2}$ Valve replacement is often required for severe regurgitation $;^{7}$ but in this patient spontaneous improvement occurred and was documented by cardiac catheterisation. As far as we are aware the left ventricular/ right atrial communciation found at operation in case 4 has not been described before as a complication of non-penetrating trauma. Ventricular septal rupture secondary to trauma is commonly low in the septum. ${ }^{2}$ The traumatic atrial septal defect found in case 6 is a rare lesion in accident survivors: most examples have been found at necropsy. ${ }^{3}$

Cardiac trauma may be missed after road accidents. In none of these six cases was the cardiac damage recognised immediately after the accident. This delayed appearance of cardiac damage has not been commented upon in previous reports though late presentation of aortic rupture as an aneurysm is well recognised. ${ }^{8}$ Apart from some relevance to the selection of hearts from road accident victims for transplantation, these findings suggest the traumatic origin of a cardiac lesion may not be appreciated. In the cases described here the cardiac abnormality was linked to an injury because the accidents were severe and the patients were young or developed a lesion which was unlikely to have any other aetiology. In older patients the defects might be ascribed to other causes, particularly if the chest trauma was not severe and had occurred several months before. Vigilance is required after road accidents to ensure that cardiac 
damage is not missed because more superficial injuries have healed.

\section{References}

${ }^{1}$ Barber $\mathbf{H}$. The effects of trauma, direct or indirect, on the heart. $Q J$ Med 1944;13:137-67.

${ }^{2}$ Liedtke AJ, DeMuth WE. Non-penetrating cardiac injuries: a collective review. Am Heart J 1973;86:687-97.

3 Parmley LF, Manion WC, Mattingly TW. Non-penetrating traumatic injury to the heart. Circulation 1958;18:371-96.

4 Symbas PN. Cardiac trauma. Am Heart J 1976;92:387-96.

${ }^{5}$ Warburg E. Myocardial and pericardial lesions due to non-penetrating injury. Br Heart $J$ 1940;2:271-80.

${ }^{6}$ East T. Traumatic rupture of the interventricular septum. Br Heart J 1945;7:116-8.

${ }^{7}$ Morgan JR, Forker AD. Isolated tricuspid insufficiency. Circulation 1971;43:559-64.

${ }^{8}$ Parmley LF, Mattingly TW, Manion WC, Jahnke EJ. Nonpenetrating traumatic injury of the aorta. Circulation 1958;17:1086-101.

\section{The International Society for Heart Transplantation}

This Society has recently been founded and will be publishing a Bulletin called Heart Transplantation. For further information please contact Dr JG Losman MD, Michael Reese Hospital and Medical Center, 29th Street and Ellis Avenue, Chicago, Illinois 60616, USA. 